\title{
Un Estudio Longitudinal de la Reputación Social no Conformista y la Violencia en Adolescentes desde la Perspectiva de Género
}

\section{A Longitudinal Study of non-Conforming Social Reputation and Violence in Adolescents From the Gender Perspective}

\author{
David Moreno Ruiz ${ }^{1}$, Saul Neves de Jesus ${ }^{2}$, Sergio Murgui Pérez ${ }^{3}$ y Belén Martínez Ferrer ${ }^{1}$ \\ ${ }^{1}$ Universidad Pablo de Olavide de Sevilla, España \\ ${ }^{2}$ Universidade do Algarve, Portugal \\ ${ }^{3}$ Universitat de València, España
}

\begin{abstract}
Resumen. El objetivo del presente estudio fue analizar la relación existente entre la reputación social del adolescente -percibida e ideal-, y la violencia instrumental -manifiesta y relacional- en adolescentes desde una perspectiva de género. La muestra utilizada en este estudios fue de 1319 adolescentes -T1- de ambos sexos (53\% chicas y $47 \%$ chicos) y de edades comprendidas entre los 11 y los 16 años (edad media 13.5; desviación típica 1.5), escolarizados en siete centros educativos de enseñanza secundaria y, de 554 estudiantes -T2- (54\% chicas y 46\% chicos). Los instrumentos utilizados fueron la Escala de Reputación Social no conformista de Carroll, Houghton, Hattie y Durkin (1999) y la Escala de Conducta Violenta Instrumental de Little, Henrich, Jones y Hawley (2003). A partir del análisis de los modelos de ecuaciones estructurales se obtuvo que la reputación ideal y percibida no conformista (T1) se relaciona con la reputación percibida (T2). También se observó una relación indirecta entre la reputación percibida (T1) y la reputación percibida (T2) a través de la violencia manifiesta instrumental. Se analizan estos resultados desde la perspectiva de género.

Palabras clave: adolescencia, reputación social no conformista -ideal y percibida-, violencia instrumental -manifiesta y relacional-.
\end{abstract}

\begin{abstract}
The aim of the current study was to analyze the relationship between non-conformist social reputation (perceived and ideal) and instrumental violence (overt and relational) in adolescents from the gender perspective. The sample was composed of 1319 adolescents -T1- aged from 11 to 16 years old (Mean 13.5; S.D. 1.5) and both sexes (53\% girls and $47 \%$ boys) in secondary school, and 554 students -T2- (54 $\%$ girls and $46 \%$ boys). Measures were Non-conformist social reputation scale (Carroll, Houghton, Hattie and Durkin, 1999) and Instrumental violence behavior scale (Little, Henrich, Jones and Hawley, 2003). From structural equation models it was found that the non-conforming social reputation -ideal and perceived- (T1) was related with non-conforming social reputation (T2). An indirect relation was also observed between perceived reputation (T1) and perceived reputation (T2) through overt instrumental violence. Results were analyzed from a gender perspective.

Keywords: adolescence, instrumental violence (overt and relational), non-conforming social reputation (ideal and perceived).
\end{abstract}

En la presente investigación se pretende profundizar en el análisis de las relaciones existentes entre la reputación social y la violencia escolar en adolescentes desde la perspectiva de género en dos paneles temporales. Más concretamente, nos centraremos en la influencia que tienen las distintas formas de violencia, manifiesta $v s$ relacional, en el mantenimiento y mejora de la reputación social no conformista en el grupo de iguales adolescentes en un intervalo de nueve meses.

Correspondencia: David Moreno Ruiz. Universidad Pablo de Olavide de Sevilla, Departamento de Ciencias Sociales Edificio No 11 , Conde de Aranda. Ctra. de Utrera, Km. 1- 41013 Sevilla. E-mail: dmorrui@upo.es
También se considerará la perspectiva de género en la medida en que en trabajos previos se han señalado diferencias significativas entre chicos y chicas en la relación entre violencia y reputación social (Buelga, Musitu y Murgui, 2009; Buelga, Musitu, Murgui y Pons, 2008; Moreno, Estévez, Musitu y Murgui, 2009; Rose, Swenson y Waller, 2004).

En la actualidad se observa un creciente interés por el estudio de la reputación social debido al importante rol que desempeña en las relaciones del adolescente con su entorno, especialmente con su grupo de iguales. De hecho, uno de los cambios sociales más destacados en la adolescencia y que determina, en gran medida, el bienestar y la satisfacción vital es la impor- 
tancia que adquiere el grupo de iguales en el ajuste psicosocial del individuo (Buelga et al., 2009; Buelga et al., 2008; Hartup, 1996; Larson y Richards, 1994; Martínez, Moreno, Amador y Orford, 2011; Moreno et al., 2009).

Se puede definir la reputación como el producto resultante de una serie de procesos sociales esenciales, que configuran la identidad personal a través de la imagen que recibe el ser humano de los agentes significativos con los que interactúa. Es un proceso continuo de percepción-valoración del grupo de referencia sobre el individuo, que determina el grado de integración o rechazo y afecta a la autopercepción, a la autoevaluación y, también, regula su comportamiento.

A partir de esta definición, los adolescentes construyen su identidad a través de la imagen que reciben de sí mismos de las personas significativas con las que interactúan, principalmente de su grupo de compañeros y amigos. En este sentido, el grupo de iguales proporciona al adolescente la información sobre la que fundamentar su autopercepción social y física, además de incidir en los valores a través de los cuales se interpreta dicha información (Cava y Musitu, 2000). Este feedback propiciado por el grupo de referencia, determinará en buena medida la autopercepción social de la reputación del adolescente, es decir la reputación social percibida o percepción reputacional, construida fundamentalmente a partir de las reacciones del grupo de referencia, y la reputación ideal o, dicho de otro modo, la reputación que desearía proyectar entre sus iguales (Carroll, Houghton, Hattie y Durkin, 2001). Este proceso autoperceptivo es de vital importancia en la conducta y las relaciones que se establecen entre adolescentes (Buelga et al., 2009).

En este sentido, la investigación sobre la reputación social no sólo se circunscribe al examen de su asociación con factores positivos de ajuste psicosocial, como por ejemplo la autoestima y la satisfacción vital, sino que encuentra una de sus líneas más atractivas de estudio en la explicación de conductas violentas, disruptivas y antisociales. De hecho autores tan relevantes como Emler en Reino Unido y Carroll en Australia formulan diversas explicaciones de cómo el deseo por conseguir, mejorar o mantener una alta reputación no conformista, fundamentada en la rebeldía, el respeto, el liderazgo y el poder en el grupo, puede llevar al adolescente a iniciarse en una trayectoria delictiva (Carroll, Green, Houghton y Wood, 2003; Carroll et al., 2001; Emler, 2009; Emler y Reicher, 2005).

Según la teoría de la Mejora de la reputación por Objetivos (Carroll et al., 2001), algunos adolescentes presentan un alto riesgo de implicarse en conductas de abuso, pero aún no han adquirido la condición de agresor. Estos adolescentes se encuentran en un estado de transición en el cual los niveles de compromiso relacionados con los objetivos de desarrollo, propios de su edad, están disminuyendo y comienzan a comprome- terse con otro tipo de objetivos caracterizados por una actitud comportamental violenta y disruptiva, que les resulta más atractiva y que puede reportarles mayores beneficios en términos de estatus reputacional. Además, en contra de lo que a priori pueda pensarse, los adolescentes desviados presentan un alto compromiso a la hora de construir y mantener su reputación social, seleccionando y eligiendo objetivos muy específicos que les ayuden a tal fin. De tal modo que, en efecto, los adolescentes agresivos son en numerosas ocasiones figuras importantes en su grupo de iguales (Gifford-Smith y Brownell, 2003; Hawley y Vaughn, 2003) e incluso populares y queridos entre sus compañeros (Salmivalli, 1998; Torregrosa, Ingles y GarciaFernandez, 2011).

Para explorar esta asociación entre la violencia y la reputación se utiliza la clasificación de violencia elaborada por Little. Este autor, con el objetivo de facilitar la investigación sobre el comportamiento realiza una doble distinción que hace referencia tanto a su forma, manifiesta versus relacional, como a las funciones que desempeña, ofensiva, reactiva e instrumental (Little, Brauner, Jones, Nock y Hawley, 2003a; Little, Henrich, Jones y Hawley, 2003b).

Más específicamente y respecto de su forma, Little et al. (2003a, 2003b) señalan que la violencia manifiesta hace referencia a comportamientos que implican una confrontación directa hacia otros con la intención de causar daño, mientras que la violencia relacional se define como aquel acto que se dirige a provocar un daño en el círculo de amistades de otra persona o bien en su percepción de pertenencia a un grupo. Respecto de las funciones de la violencia, podemos distinguir entre la acción ofensiva, que implica una conducta violenta sin mediar un estimulo desencadenante de la agresión, la acción reactiva que supone una respuesta defensiva ante alguna provocación y, por último, la acción instrumental, que implica un comportamiento deliberado de anticipación de beneficios y que está controlado por refuerzos externos (Little et al. 2003a, 2003b).

La mayoría de los estudios realizados sobre reputación social y comportamiento violento, que utilizan la clasificación propuesta por Little analizan sólo violencia manifiesta o relacional, y aúnan las tres funciones de la violencia en un mismo factor (Buelga et al., 2009; Buelga et al., 2008; Moreno et al., 2009). Sin embargo, para la elaboración del presente estudio se han seleccionado los dos tipos de violencia respecto a su forma -manifiesta y relacional-, y la dimensión instrumental respecto a su función. Además, y como novedad, las variables de violencia seleccionadas, violencia manifiesta instrumental y violencia relacional instrumental, son analizadas independientemente en su asociación con la reputación social no conformista. El interés de este objetivo reside en analizar la relación existente entre la reputación social percibida de los adolescentes evaluada en tiempo 1 (T1) con los distintos tipos de conducta violenta instrumental (manifiesta y relacio- 
nal), y, el desarrollo y mantenimiento de la reputación percibida no conformista medida en tiempo 2 (T2). Del mismo modo se pretende profundizar en estás relaciones en función del género.

Teniendo en cuenta lo anteriormente expuesto, proponemos las siguientes hipótesis: (1) la reputación ideal no conformista y la reputación percibida no conformista en T1 se relacionarán directa y positivamente con la reputación percibida no conformista en T2; (2) la reputación percibida no conformista en T1 se relacionará directamente con la violencia manifiesta instrumental y la violencia relacional instrumental, y ambas se relacionarán, a su vez, con una alta reputación percibida en T2. La relación planteada en la hipótesis 2 será significativamente distinta en función del género. De manera más detallada: (3) la relación indirecta entre la reputación percibida en T1 y la reputación percibida en T2 en los chicos se relacionará, fundamentalmente, a través de la violencia manifiesta instrumental, mientras que en el caso de las chicas esta relación estará mediada, principalmente, por la violencia relacional.

\section{Método}

\section{Muestra}

A principios del mes de Octubre (T1) se realizó la primera administración de los cuestionarios. En este T1 participaron un total de 1319 adolescentes de ambos sexos (53\% chicas y $47 \%$ chicos) y edades comprendidas entre los 11 y los 16 años (edad media 13.5; desviación típica 1.5), escolarizados en siete centros educativos de enseñanza secundaria ubicados en la Comunidad Valenciana (provincias de Alicante y Valencia) y distribuidos en $1^{\circ}, 2^{\circ}, 3^{\circ}$ y $4^{\circ}$ de la ESO y $1^{\circ}$ y $2^{\circ}$ de Bachillerato. Durante el mes de Junio se realizó una segunda aplicación de los cuestionarios. En este T2, tres centros escolares rehusaron continuar en este estudio. La muestra final quedó conformada por 554 estudiantes, de los cuales el $46 \%$ fueron chicos (257) y el $54 \%$ chicas (297).

\section{Instrumentos}

Escala de Reputación Social no conformista. Este instrumento se compone de las subescalas autopercepción de la reputación y self público ideal reputacional de la Escala de Reputación Social elaborada por Carroll, Houghton, Hattie y Durkin (1999; traducción bidireccional inglés-español). La escala evalúa con 15 ítems y con un rango de respuesta que va de 1 -nuncaa 4 -siempre-, la percepción del adolescente de su reputación real no conformista (e.g., "Los demás piensan que soy un/a chico/a rebelde") y de su reputación ideal no conformista (e.g., "Me gustaría que los demás pensasen que soy un/a chico/a rebelde"). La consistencia interna medida a través del alfa de Cronbach para ambas subescalas fue de .85 y .78 respectivamente.

Escala de Conducta Violenta Instrumental. Utilizamos para el presente estudio las subescalas de violencia manifiesta y violencia relacional de la Escala de Conducta Agresiva de Little, Henrich, Jones y Hawley (2003; traducción bidireccional inglés-español). El instrumento mide con 9 ítems y con un rango de respuesta de 1 -mиу en desacuerdo- a 4 -mиy de acuerdo-, comportamientos que implican agresiones de carácter instrumental, como violencia manifiesta instrumental (conductas violentas físicas o verbales como medio para conseguir un fin; e.g., "Pego, doy patadas o puñetazos para conseguir lo que quiero") y violencia relacional instrumental (comportamientos que implican violencia de carácter social, como la exclusión o el aislamiento del grupo, o la manipulación de las relaciones sociales del otro como medio para conseguir un fin; e.g., "Para conseguir lo que quiero, no dejo que algunas personas formen parte de mi grupo de amigos/as"). El alfa de Cronbach obtenido para estas subescalas en nuestros datos fue de .84 y .78 respectivamente.

\section{Procedimiento}

La selección de los centros educativos se llevó a cabo de manera aleatoria entre el total de escuelas públicas de enseñanza secundaria obligatoria de la Comunidad Valenciana. En primer lugar, se envió una carta a los centros educativos seleccionados explicando el proyecto de investigación. Posteriormente se contactó telefónicamente con la dirección de los centros y se concertó una entrevista en la que se explicó el proyecto con detalle, y se entregaron los consentimientos informados para los padres y para el alumnado junto con una carta explicativa de la investigación que la dirección de los centros se encargó de tramitar. Tras la obtención de los permisos correspondientes de la dirección de los centros educativos, se realizó un seminario informativo con la plantilla de profesorado para explicar la importancia y el alcance del estudio. La aplicación de los instrumentos se llevó a cabo por un grupo de investigadores expertos y entrenados. La batería de instrumentos se administró a los adolescentes en sus aulas habituales durante un período regular de clase dónde el profesor-tutor del curso siempre estuvo presente. El orden de administración de los instrumentos se contrabalanceó en cada clase y centro educativo. Se informó en todo momento a los adolescentes que la participación en la investigación era voluntaria y anónima. A principios del mes de Octubre (T1) se realizó la primera aplicación de los instrumentos. Previamente a la administración de los cuestionarios, se informó a los adolescentes que el objetivo del estudio era conocer algunos aspectos de 
la vida escolar y de sus relaciones con profesores y compañeros. Se les informó del anonimato de sus respuestas y se les insistió en la importancia de su sinceridad.

Durante el mes de Junio, se volvió a contactar con los mismos centros escolares para realizar una segunda administración de los cuestionarios. En este segundo momento (T2), tres centros escolares rehusaron continuar en este estudio a causa de la excesiva saturación de tareas que tenía el profesorado en ese momento por la cercanía del final del curso y su preocupación por el cumplimiento de sus objetivos académicos. El estudio cumplió los valores éticos requeridos en la investigación con seres humanos, respetando los principios fundamentales incluidos en la Declaración Helsinki, en sus actualizaciones, y en las normativas vigentes (consentimiento informado $y$ derecho a la información, protección de datos personales y garantías de confidencialidad, no discriminación, gratuidad y posibilidad de abandonar el estudio en cualquiera de sus fases). yeron en el cálculo del modelo de ecuaciones estructurales.

A continuación, se puso a prueba un modelo de ecuaciones estructurales con el programa EQS 6.0 (Bentler, 1995) para analizar la relación existente entre las variables. Para determinar la bondad de ajuste del modelo y la significación estadística de los coeficientes, se utilizaron estimadores robustos debido a la desviación de la normalidad de los datos (coeficiente Mardia normalizado: 18.44). El modelo calculado ajustó bien a los datos como indican los siguientes índices: $\mathrm{CFI}=.99, \mathrm{IFI}=$ $.99, \mathrm{NNFI}=.99$, y RMSEA $=.038$. Para los índices CFI, IFI y NNFI se consideran aceptables valores superiores a .95 , y para el índice RMSEA valores inferiores a .05 (Batista y Coenders, 2000). Finalmente, para comprobar si las relaciones observadas en el modelo general difieren según el género, se calcularon de manera independiente un modelo de ecuaciones estructurales para chicos y otro para chicas.

Los factores incluidos en el modelo fueron: (1) reputación percibida no conformista medida en T1; (2)

Tabla 1. Correlaciones de Pearson entre las variables para el modelo general

\begin{tabular}{|c|c|c|c|c|c|}
\hline Variables & 1 & 2 & 3 & 4 & 5 \\
\hline 1. Violencia manifiest instrumental & - & & & & \\
\hline 2. Violencia relacional instrumental & $.65 * *$ & - & & & \\
\hline 3. Reputación percibida (T1) & $.36 * *$ & $.22 * *$ & - & & \\
\hline 4. Reputación ideal & $.20 * *$ & $.20 * *$ & $.58 * *$ & - & \\
\hline 5. Reputación percibida (T2) & $.36 * *$ & $.20 * *$ & $.49 * *$ & $.35^{* *}$ & - \\
\hline
\end{tabular}

Nivel de significación: $* * p<0.01$

\section{Resultados}

En primer lugar se calcularon las correlaciones de Pearson entre todas las variables objeto de estudio para el modelo general en la Tabla 1, así como para las correlaciones por género en la Tabla 2. Los resultados muestran correlaciones significativas entre todas las variables en el sentido esperado, por lo que se inclu- reputación ideal no conformista; (3) conducta violenta manifiesta instrumental; (4) conducta violenta relacional instrumental y (5) reputación percibida no conformista medida en $\mathrm{T} 2$.

El modelo explica el $28.5 \%$ de la varianza de la reputación social no conformista medida en T2, lo que parece evitar un posible error de tipo II (García, Pascual, Frías, Van Krunckelsven y Murgui, 2008).

Tabla 2. Correlaciones de Pearson entre las variables para los modelos de chicos y chicas ${ }^{1}$

\begin{tabular}{|c|c|c|c|c|c|}
\hline Variables & 1 & 2 & 3 & 4 & \\
\hline 1. Violencia manifiesta instrumental & - & & & & \\
\hline 2. Violencia relacional instrumental & $\begin{array}{l}.64 * * \\
.64 * *\end{array}$ & - & & & \\
\hline 3. Reputación percibida (T1) & $\begin{array}{l}.39 * * \\
.33 * *\end{array}$ & $\begin{array}{l}.21^{* *} \\
.24 * *\end{array}$ & - & & \\
\hline 4. Reputación ideal & $\begin{array}{l}.22 * * \\
.19 * *\end{array}$ & $\begin{array}{l}.22 * * \\
.18 * *\end{array}$ & $\begin{array}{l}.57 * * \\
.59 * *\end{array}$ & $\begin{array}{l}- \\
-\end{array}$ & \\
\hline 5. Reputación percibida (T2) & $\begin{array}{l}.44 * * \\
.28 * *\end{array}$ & $\begin{array}{l}.20 * * \\
.21 * *\end{array}$ & $\begin{array}{l}.46^{* *} \\
.52^{* *}\end{array}$ & $\begin{array}{l}.33^{* *} \\
.36^{* *}\end{array}$ & $\begin{array}{l}- \\
-\end{array}$ \\
\hline
\end{tabular}

Nivel de significación: $* * p<0.01$

${ }^{1}$ El índice de correlación superior pertenece a los chicos y el inferior a las chicas. 
Figura 1. Modelo estructural final general ${ }^{1}$

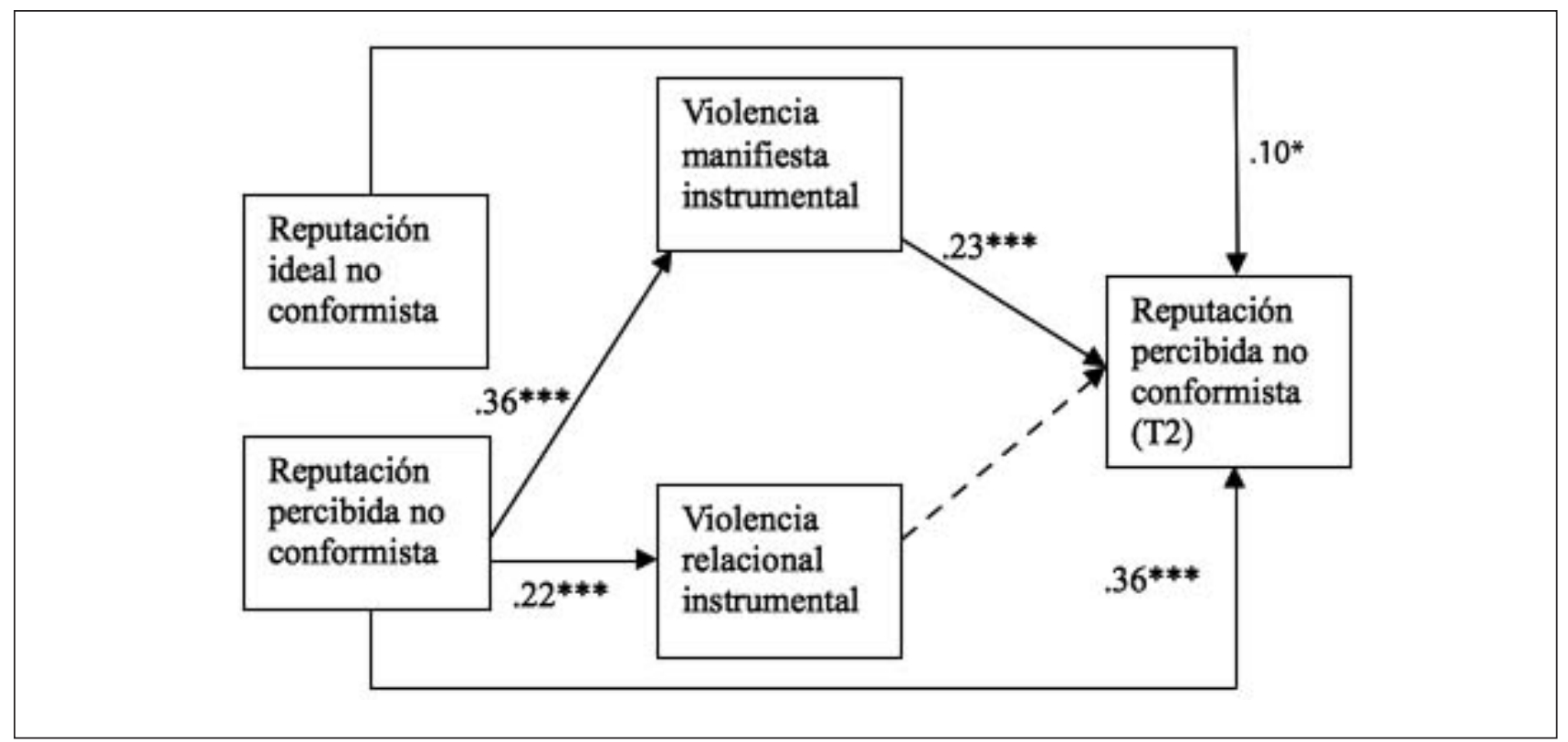

${ }^{1}$ Las flechas unidireccionales representan relaciones de influencia significativas entre variables. La significación de las relaciones se ha determinado a partir del error estándarrobusto: nivel de significación.

$p<.05 ; * * * p<.001$

La Figura 1 muestra la representación gráfica del modelo estructural final general con los coeficientes estandarizados y su probabilidad asociada.

En esta figura se observan, en primer lugar, las distintas relaciones de influencia entre la reputación percibida medida en T1 y T2, la reputación ideal y las distintas violencias instrumentales del adolescente en la escuela. La reputación ideal presentó una relación directa y positiva con la reputación percibida no conformista medida en T2 $(b=.10, p<.05)$. La reputación percibida no conformista en T1 mostró una relación directa y positiva con la reputación percibida no conformista en T2 $(b=.36, p<.001)$, con la violencia manifiesta instrumental y con la violencia relacional instrumental $(b=.36, p<.001$ y $b=$ $.22, p<.001$, respectivamente). Además la reputa-

Figura 2. Modelo estructural final para chicos ${ }^{1}$

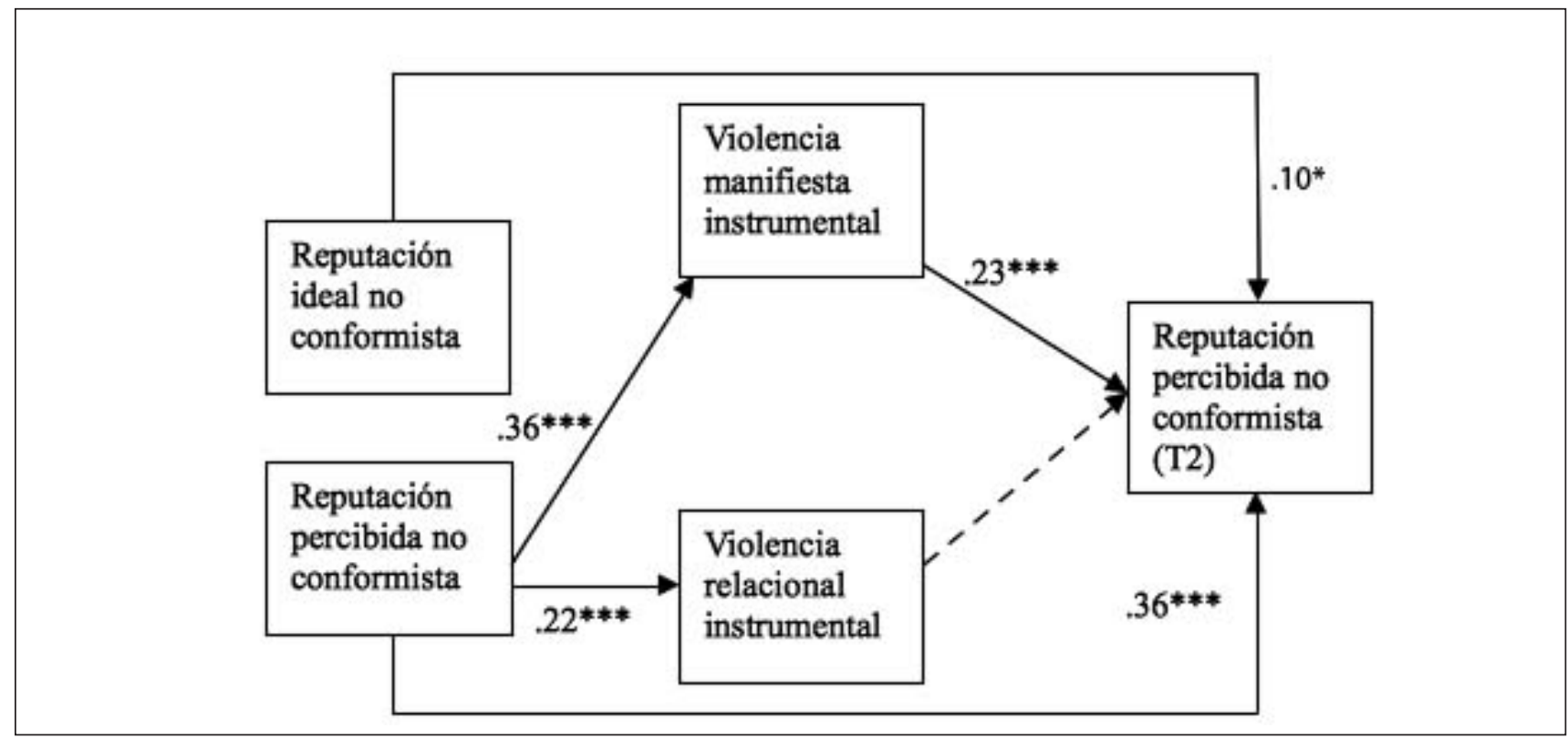

${ }^{1}$ Las flechas unidireccionales representan relaciones de influencia significativas entre variables. La significación de las relaciones se ha determinado a partir del error estándar robusto: nivel de significación.

$* p<.05 ; * *<<.001 ; * * * p<.001$ 
Figura 3. Modelo estructural final para chicas ${ }^{1}$

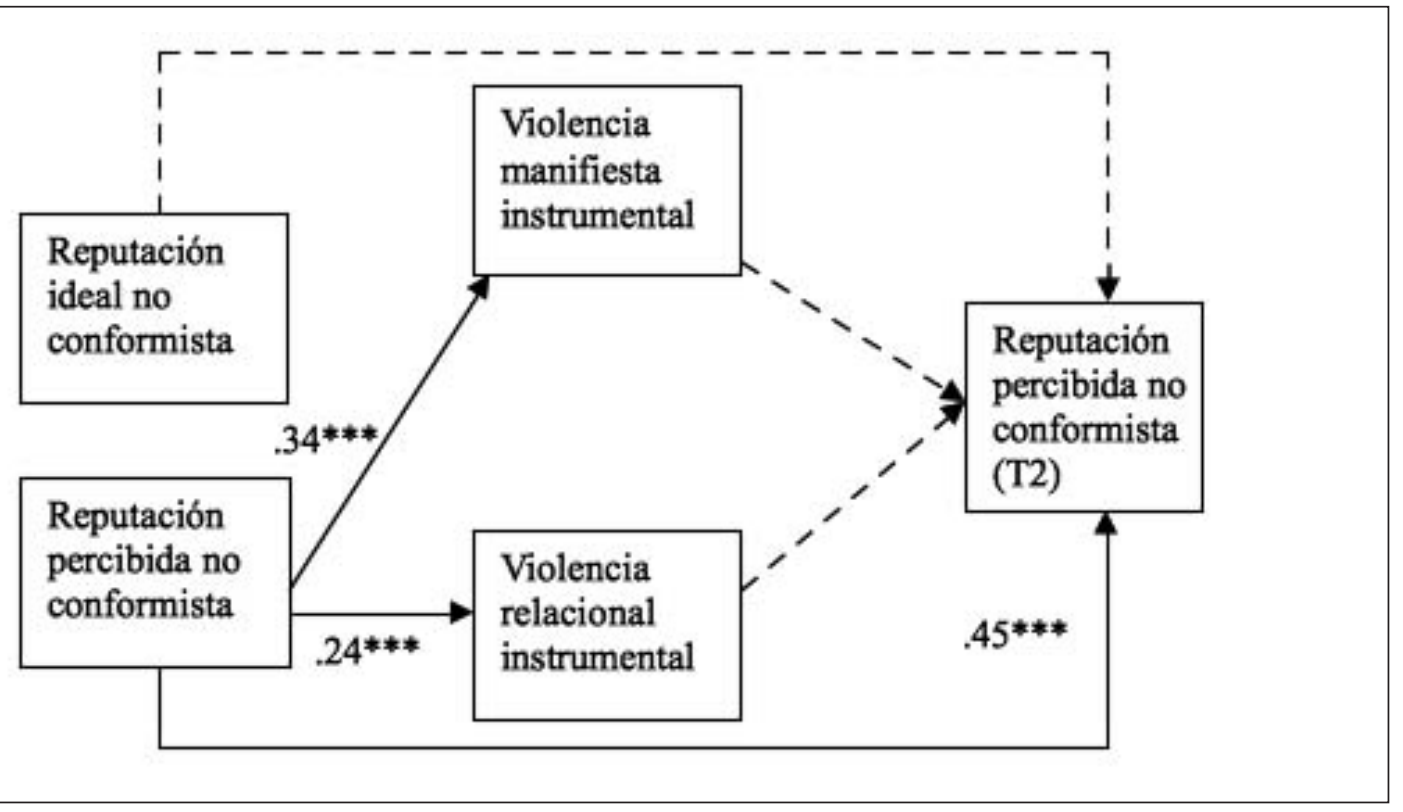

${ }^{1}$ Las flechas unidireccionales representan relaciones de influencia significativas entre variables. La significación de las relaciones se ha determinado a partir del error estándar robusto: nivel de significación.

$* * * * p<.001$

ción percibida en T1 también presentó una relación indirecta con la reputación percibida en T2 pero sólo a través de la violencia manifiesta instrumental $(b=$ $.23, p<.001)$. La relación entre violencia relacional instrumental y reputación percibida en T2 no fue significativa.

Respecto a los análisis por género, es importante destacar que, a diferencia de lo que sucede en el modelo general, en los modelos de ecuaciones estructurales calculados de manera independiente para chicos y chicas la relación entre reputación ideal y reputación percibida en T2 no fue significativa. Los resultados obtenidos en el caso de los chicos mostraron una relación directa y positiva entre la reputación percibida en T1 y la reputación percibida en T2 $(b=.26$, $p<.001)$, no obstante esta asociación resultó ser más fuerte en el modelo calculado para las chicas $(b=.45$, $p<.001)$. Además, en el modelo calculado para los chicos se observó una relación indirecta entre la reputación percibida no conformista en $\mathrm{T} 1$ y la reputación percibida en T2 a través de las variables violencia manifiesta instrumental y violencia relaciona instrumental. Sin embargo esta asociación fue positiva para la violencia manifiesta y negativa para la violencia relacional $(b=.37, p<.001$ y $b=-.13, p<.001$, respectivamente). En el caso de las chicas la relación indirecta entre reputación percibida en $\mathrm{T} 1$ y reputación percibida en T2 no fue significativa. Finalmente se puede apuntar que, aún sin ser estadísticamente significativa, la relación entre violencia relacional instrumental y la reputación percibida en T2 fue positiva, lo contrario que sucedió en los chicos.

\section{Discusión y Conclusiones}

En relación con la primera hipótesis se ha observado que la reputación ideal y la reputación percibida en T1 se relacionan directa y positivamente con la reputación percibida no conformista en T2, con lo cual se confirma la hipótesis planteada. No obstante, un aspecto destacable en estas relaciones es que al analizarlas en chicos y en chicas se constató en ambos casos la relación entre reputación percibida en T1 y la reputación en T2. Sin embargo, no se confirmó la relación entre la reputación ideal en T1 y la reputación percibida en T2. Estos resultados no eran los esperados, en la medida en que está abundantemente demostrado que las relaciones entre ambas dimensiones es significativa; sin embargo al introducir un intervalo temporal de nueve meses se observó que las relaciones no fueron las previsibles. Estos datos, que aparentemente no son relevantes, consideramos que tienen una especial importancia. Por tanto, ante esta situación no esperada, nos realizamos la siguiente pregunta: ¿qué ha sucedido en esta relación para que en el transcurso de nueve meses la reputación ideal no conformista no tenga relaciones con la reputación percibida no conformista? En otros trabajos en los que se fundamenta esta investigación se ha constatado las relaciones existentes entre estas dos dimensiones pero ninguno de estos trabajos se ha analizado incorporando un intervalo temporal (Buelga et al., 2009; Buelga et al., 2008; Carroll et al., 2003; Carroll et al., 2001; Moreno et al., 2009). Creemos que, por la relevancia de estas variables, este hallazgo merecería una mayor investigación o seguir 
explorando estas relaciones y comprobar su estabilidad. Este resultado puede deberse a los procesos psicosociales relacionados con los iguales y el profesor que han acontecido durante los nueve meses en este escenario y que se sucedieron en el transcurso de esta investigación.

En relación con la segunda hipótesis se observó que la reputación percibida no conformista (T1) se relacionaba con la reputación percibida (T2) a través de la violencia manifiesta instrumental de forma positiva. Sin embargo, la violencia relacional instrumental no se relacionó con la reputación percibida (T2), con lo cual la hipótesis se confirma parcialmente, en la medida en que se preveía que la reputación percibida se relacionaría con la reputación en T2 a través de la violencia relacional instrumental. Este resultado contrasta con los obtenidos en otros trabajos en los que se han analizado estas relaciones (Cillessen y Mayeux, 2004; Nelson, Robinson y Hart, 2005; Rose et al., 2004). Creemos que esta divergencia podría atribuirse al hecho de que el modelo general se ha construido con chicos y chicas, y parece que existe una gran consistencia entre los resultados hallados entre los investigadores en atribuir a los hombres una mayor implicación en conductas violentas, especialmente manifiestas, que a las mujeres a las que normalmente se les ha atribuido comportamientos violentos de carácter relacional (Cerezo y Ato, 2011; Crick, Casas y Nelson, 2002; Crick y Grotpeter, 1996; Postigo, González, Mateu, Ferrero y Martorell, 2009; Povedano, Estévez, Martínez y Monreal, 2011). Este aspecto lo analizaremos a continuación puesto que los resultados de este estudio coinciden en parte con esta idea.

Respecto de la tercera hipótesis se ha constatado que la reputación percibida en T1 se relaciona con la reputación percibida en $\mathrm{T} 2$, principalmente, a través de la violencia manifiesta instrumental. También, se observó una asociación, aunque más débil, entre violencia relacional instrumental y reputación percibida. En este sentido, consideramos de interés subrayar que la relación entre reputación percibida en T1 y violencia relacional fue positiva, pero, al contrario de lo esperado la asociación entre este tipo de violencia y la reputación percibida en T2 fue negativa. Este resultado es muy sugerente para explicar las diferencias entre chicos y chicas desde la perspectiva de género. Son numerosos los trabajos en los que se han obtenido resultados similares en la asociación entre violencia manifiesta y reputación social, en el sentido de que los chicos utilizan la confrontación directa y las conductas de violencia física con más frecuencia que las chicas, normalmente, para obtener un mayor poder en el grupo de iguales (Cerezo y Ato, 2011; Hoff, Reese-Weber, Schneider y Stagg, 2009; Villarreal-González, Sánchez-Sosa, Veiga y del Moral-Arroyo, 2011).

En esta situación, una vez que los chicos han obtenido un alto estatus no conformista continúan ejerciendo este tipo de comportamientos violentos manifiestos para mantener o mejorar la posición que han adquirido en el grupo. Sin embargo, es posible que ocurra lo contrario con el uso de la violencia relacional. Es decir, los chicos que tienen un comportamiento rebelde y violento pueden ejercer distintos actos de violencia relacional pero lejos de proporcionarles un mayor estatus reputacional parece, según los datos obtenidos, que este tipo de violencia relacional menoscaba su posición en el grupo de iguales. Se podría pensar que lo que subyace en esta relación son los estereotipos de género vigentes en nuestra sociedad en la que se valora de forma más positiva en los chicos el enfrentamiento directo más que el urdir tramas o enfrentarse indirectamente con los demás para conseguir un fin, que parece más vinculado al género femenino (Rose et al., 2004; Snethen y Van Puymbroeck, 2008). Este es un aspecto que, por su novedad e interés en la explicación de la violencia en función del género, merecería una mayor exploración.

En el caso de las chicas no se cumple la hipótesis planteada, ya que se observó una relación positiva más alta entre reputación percibida en $\mathrm{T} 1$ con la violencia manifiesta instrumental que con la violencia relacional, aunque esta también es significativa. En trabajos previos se ha comprobado que las motivaciones relacionales y el desarrollo de habilidades sociales de manera más tardía que en sus iguales de género, son una causa importante en la expresión de la violencia, física y relacional y en el desarrollo de trastornos de conducta en las chicas (ABA y NBA, 2001; Keenan y Shaw, 1997).

También, los resultados obtenidos en esta investigación son convergentes con los de otros trabajos más actuales que observaron un incremento de las conductas violentas manifiestas en chicas, aún siendo estas más frecuentes en los chicos (Bowie, 2007). Estos resultados podrían atribuirse al hecho de que las intervenciones llevadas a cabo en este ámbito con chicas, se han centrado de forma predominante en la reducción de la violencia relacional, generalmente asociada al género femenino (Snethen y Van Puymbroeck, 2008), y soslayando la violencia física en estos programas. Igualmente, se ha detectado que la relación entre reputación y violencia relacional es más fuerte en el caso de las chicas que en el de los chicos, tal y como se observó en anteriores trabajos (Moreno et al., 2009).

Curiosamente, un resultado que no habíamos previsto en esta investigación ha sido la ausencia de relación entre las dos violencias analizadas, manifiesta y relacional, y la reputación social en T2. Se podría interpretar este resultado no esperado al hecho de que las chicas que tienen una imagen de si misma como persona dura y rebelde se implican con mayor frecuencia en actos violentos manifiestos y relacionales, lo cual parece que no tiene una influencia en la posterior autopercepción de su reputación, lo contrario de lo que sucede en los chicos.

Finalmente, aunque este estudio se fundamenta en el análisis de dos paneles temporales con un intervalo de 
nueve meses, sería interesante seguir explorando estas relaciones para enriquecer y validar la información obtenida en esta investigación. Del mismo modo que otros autores, consideramos importante que se preste la atención adecuada al estudio de la conducta violenta en chicas (Chesney-Lind y Belknap, 2004), al igual que sucede con la investigación de la violencia en chicos. Las chicas pueden expresar violencia física o manifiesta por distintos motivos que los chicos, y por tanto, tener distintas motivaciones lo cual exige el diseño, desarrollo y evaluación de programas psicoeducativos y de intervención que tengan en cuenta el género de los participantes (ABA y NBA, 2001; Acoca, 1998). Estos programas psicoeducativos en el contexto escolar deben promocionar las estrategias para prevenir y resolver las situaciones de indisciplina y violencia en la escuela (Jesus, 2001; Pereira, 2006).

\section{Referencias}

Acoca, L. (1998). Outside/inside: The violation of American girls at home, on the street, and in the juvenile justice system. Crime \& Delinquency, 44 (4), 561-589.

American Bar Association y National Bar Association (2001). Justice by gender: The lack of appropriate prevention, diversion, and treatment alternatives for girls in the justice system. Washington DC: Author.

Bentler, P. M. (1995). EQS structural equations program manual. Encino, CA: Multivariate Software.

Bowie, B. H. (2007). Relational aggression, gender and the devolpmental process. Journal of Child and Adolescent Psychiatric Nursing, 20, 107-115.

Buelga, S., Musitu, G. y Murgui, S. (2009). Relaciones entre la reputación social y la agresión relacional en la adolescencia. International Journal of Clinical and Health Psychology, 9 (1), 127-141.

Buelga, S., Musitu, G., Murgui, S. y Pons, J. (2008). Reputation, loneliness, satisfaction with life and aggressive behavior in adolescence. The Spanish Journal of Psychology, 11, 192-200.

Carroll, A., Green, S., Houghton, S. y Wood, R. (2003). Reputation enhancement and involvement in delinquency among high school students. International Journal of Disability, Development and Education, 50, 253-273.

Carroll, A., Houghton, S., Hattie, J. y Durkin, K. (1999). Adolescent reputation enhancement: differentiating delinquent, nondelinquent, and at-risk youths. Journal of Child Psychology and Psychiatry, 40, 593-606.

Carroll, A., Houghton, S., Hattie, J. y Durkin, K. (2001) Reputation Enhancing Goals: Integrating Reputation Enhancement and Goal Setting Theory as an Explanation of Delinquent Involvement. En Columbus, F. H. (Ed). Advances in Psychology Research, (pp. 101-129). New York: Nova Science Publishers.

Cava, M. J., y Musitu, G. (2000). La potenciación de la autoestima en la escuela. Barcelona: Paidós.

Cerezo, F. y Ato, M. (2010). Social status, gender, classroom climate and bullying among adolescents pupils. Anales de Psicología, 26 (1), 137-144.

Chesney-Lind, M. y Belknap, J. (2004). Trends in delinquent girls' aggression and violent behavior: A review of the evidence. En M. Putzllaz \& K.L. Bierman (Eds.), Aggression, antisocial behavior, and violence among girls: A developmental perspective. New York: Guilford Press.

Cillessen, A. H. N. y Mayeux, L. (2004). From censure to reinforcement: Developmental changes in the association between aggression and social status. Child Development, 75, 147-163.

Crick, N. R., Casas, J. F. y Nelson, D.A. (2002). Toward a more comprehensive understanding of peer maltreatment: Studies of relational victimization. Current Directions in Psychological Science, 11, 98-101.

Crick, N. R. y Grotpeter, J. K. (1996). Children's treatment by peers: Victims of relational and overt aggression. Development and Psychopathology, 8, 367-380.

Emler, N. (2009). Delinquents as a minority group: Accidental tourists in forbidden territory or voluntary émigrés? En F. Butera \& J. Levine (Eds.), Coping with minority status: Responses to exclusion and inclusion. Cambridge: Cambridge University Press.

Emler, N. y Reicher, S. (2005). Delinquency: Cause or consequence of social exclusion? En D. Abrams, M.A. Hogg y J. M. Marques (Eds.), The Social Psychology of Inclusion and Exclusion (pp. 211-241). New York: Psychology Press.

García, J. F., Pascual, J., Frías, M. D., Van Krunckelsven, D. y Murgui, S. (2008). Diseño y análisis de la potencia: n y los intervalos de confianza de las medias. Psicothema, 20, 933-938.

Gifford-Smith, M. E. y Brownell, C. A. (2003). Childhood peer relationships: social acceptance, friendships, and social network. Journal of School Psychology, 41, 235-284.

Hartup, W. W. (1996). The company they keep: Friendships and their developmental significance. Child Development, 67, 1-13.

Hawley, P. y Vaughn, B. (2003). Aggression and adaptation: The bright side to bad behavior. Introduction to special volume. Merrill-Palmer Quarterly, 49, 239-244.

Hoff, K. E., Reese-Weber, M., Schneider, W. J. y Stagg, J. W. (2009). The association between high status positions and aggressive behavior in early adolescence. Journal of School Psychology, 47, 395-426.

Jesus, S. N. (2001). Como prevenir o stress e resolver a indisciplina dos alunos? Porto: ASA Editores.

Keenan, K. y Shaw, D. (1997). Developmental and social influences on young girls' early problem behavior. Psychological Bulletin, 121, 95-113.

Larson, R. y Richards, M. H. (1994). Divergent realities: The emotional lives of fathers, mothers, and adolescents. New York: Basic Books.

Little, T., Brauner, J., Jones, S., Nock, M. y Hawley, P. (2003). Rethinking aggression: A typological examination of the functions of aggression. Merrill-Palmer Quarterly, 49, 343-369. 
Little, T., Henrich, C., Jones, S., y Hawley, P. (2003). Disentangling the "whys" from the "whats" of aggressive behaviour. International Journal of Behavioral Development, 27, 122-133.

Martínez, B., Moreno, D., Amador, L. V. y Orford, J. (2011). School Victimization Among Adolescents. An Analysis from an Ecological Perspective. Psychosocial Intervention, 20, 149-160.

Moreno, D., Estévez, E., Murgui, S. y Musitu, G. (2009). Reputación social y violencia relacional en adolescentes: el rol de la soledad, la autoestima y la satisfacción vital. Psicothema, 21, 537-542.

Nelson, D. A., Robinson, C. C. y Hart, C. H. (2005). Relational and physical aggression of preschool-age children: Peer status linkages across informants. Early Education and Development, 16, 115-139.

Pereira, B. O. (2006). Prevenção da violência em contexto escolar : diagnóstico e programa de intervenção. In J. Neto \& M. Nascimento (Eds.), Infância: violência, instituições e políticas públicas (Cap. 2, pp. 43-51). São Paulo: Expressão e Arte.

Postigo, S., González, R., Mateu, C., Ferrero, J. y Martorell, C. (2009). Diferencias conductuales según género en convivencia escolar. Psicothema, 21, 453-458.

Povedano, A., Estévez, E., Martínez, B. y Monreal, M. C. (en prensa). Un perfil psicosocial de adolescentes agresores y víctimas en la escuela: Análisis de las diferencias de género. Revista de Psicología Social.

Rose, A. L., Swenson, L. P. y Waller, E. M. (2004). Overt and relational aggression and perceived popularity: Developmental differences in concurrent and pros- pective relations. Developmental Psychology, 40, 378387.

Salmivalli, C. (1998). Intelligent, attractive, well-behaving, unhappy: the structure of adolescents' self-concept and its relations to their social behavior. Journal of Research on Adolescence, 8, 333-354.

Snethen, G. y van Puymbroeck, M. (2008). Girls and physical aggression: Causes, trends, and intervention guided by Social Learning Theory. Aggression and Violent Behavior, 13, 346-354.

Torregrosa, M. S., Ingles, C. J. y Garcia-Fernandez, J. M. (2011). Aggressive Behavior as a Predictor of SelfConcept: AStudy with a Sample of Spanish Compulsory Secondary Education Students. Psychosocial Intervention, 20, 201-212.

Villarreal-González, M. E., Sánchez-Sosa, J. C., Veiga, F. H. y del Moral-Arroyo, G. (2011). Development Contexts, Psychological Distress, Social Self-Esteem and School Violence from a Gender Perspective in Mexican Adolescents. Psychosocial Intervention, 20, 171-181.

\section{Nota de los autores}

Este estudio ha sido elaborado en el marco del proyecto de investigación PSI2008-01535/PSIC: "Violencia escolar, victimización y reputación social en la adolescencia", subvencionado por el Ministerio de Ciencia e Innovación de España, y del proyecto de investigación educativa 2009 PIV-015/09: "Violencia y victimización en la adolescencia: análisis desde una perspectiva de género", subvencionado por la Consejería de Educación de la Junta de Andalucía y los Fondos Europeos FEDER.

Manuscrito recibido: 12/04/2011

Revisión recibida: 25/08/2011

Manuscrito aceptado: 05/09/2011 